\title{
Morphological characterization using scanning electron microscopy of fly artifacts deposited by Calliphora vomitoria (Diptera: Calliphoridae) on household materials
}

\author{
Guido Pelletti ${ }^{1}$. Desiree Martini ${ }^{2} \cdot$ Laura Ingrà $^{2} \cdot$ Maria Carla Mazzotti $^{1} \cdot$ Arianna Giorgetti $^{1}$ (D) $\cdot$ Mirella Falconi $^{2}$. \\ Paolo Fais ${ }^{1}$
}

Received: 17 March 2021 / Accepted: 9 June 2021 / Published online: 1 July 2021

(C) The Author(s) 2021

\begin{abstract}
Insects found at a crime scene can produce traces referred to as fly artifacts (FA) due to their movement over the corpse and the manner in which they feed upon it. These can be detrimental for carrying out criminal investigations. Confusing a FA with a genuine bloodspot can lead to misinterpretations, also taking into consideration that FA may contain a human DNA profile. The aim of the present study was to employ scanning electron microscopy (SEM) for the analysis of FA produced by Calliphora vomitoria on hard surfaces and fabrics that are commonly present at crime scenes. FA and control bloodstains were produced under experimental conditions on metal, glass, plaster, cotton, and polyester. After macroscopic analysis, FA were examined at standard low $(20-40 \times)$, medium low $(300-600 \times)$, and high ultrastructural $(1200 \times)$ magnification through a SEM Stereoscan 360, Leica, Cambridge. SEM analysis enabled the identification of distinctive features of FA on hard surfaces, namely, amorphous crystals, micro-crystals with a morphology similar to those of uric or micro-crystals with a comparable morphology to cholesterol, absent in controls. Moreover, red blood cells (RBC) were absent in FA but were always present in controls. On cotton, for both FA and controls, the drop was almost completely absorbed and thus indistinguishable from the underlying fabric texture. On polyester, FA showed amorphous/crystal-like deposits and no RBC, as observed on hard surfaces, except for those showing a completely flat surface. SEM analysis appeared to be suitable for differential diagnosis between FA and genuine bloodstains on hard surfaces, although the results may be inconclusive on tested fabrics.
\end{abstract}

Keywords Forensic pathology $\cdot$ Crime scene $\cdot$ Bloodstain pattern analysis $\cdot$ Fly artifacts $\cdot$ Scanning electron microscopy

Guido Pelletti and Desiree Martini equally contributed and should be considered as co-first authors

Arianna Giorgetti

arianna.giorgetti@unibo.it; ari.giorgetti@gmail.com

Guido Pelletti

guido.pelletti2@unibo.it

Desiree Martini

desiree.martini@unibo.it

Laura Ingrà

laura.ingra@unibo.it

Maria Carla Mazzotti

mariacarla.mazzotti2@unibo.it

\section{Introduction}

Insects found at a crime scene can be of use to many types of forensic investigation. They can provide information about time since death, season of death, the primary crime scene, movement or concealment of the remains following

Mirella Falconi

mirella.falconi@unibo.it

Paolo Fais

paolo.fais@unibo.it

1 Department of Medical and Surgical Sciences, Unit of Legal Medicine, University of Bologna, Via Irnerio 49, 40126 Bologna, Italy

2 Department of Biomedical and Neuromotor Sciences, University of Bologna, Bologna, Italy 
death, specific sites of trauma on the body, use of drugs, and victim identification when the body is removed from the initial decomposition site [1-3]. However, the presence of insects does not always aid investigations and may even prove counterproductive by reason of their movement over the corpse and the manner in which they feed on it. Flies in the Calliphoridae and Sarcophagidae families land on the corpse or on biological fluids, walking across the surfaces and feeding. Foraging activity is known to cause the most problematic alteration of the death scene, as insects can create unique stains or intermix fly artifacts with bloodstains and other human body fluids as a result of their digestive process [4]. Insects can also create transfer patterns with the tarsi or the abdomen or leave impressions after passing through the fluids [5]. Confusing FA with genuine bloodspots can lead to misinterpretations, also considering that FA may contain human DNA profile [6].

Certain macroscopic key features may help an investigator to distinguish fly artifacts from blood spots [7, 8]. However due to the wide range of shapes, colors, and sizes and the potentially high number of FA found at crime scenes, there is a strong probability that FA can appear very similar to genuine drops of blood [5]. In such cases, the visual morphologic approach should be integrated with a confirmatory technique for identification [9].

A recent study from our group showed that scanning electron microscopy (SEM) allows the visualization of ultrastructural morphological differences between FA deposited by Sarcophaga carnaria and blood controls on different types of paper - a promising tool for performing a differential diagnosis between FA and bloodstains [10].

However, the main problem associated with visual or ultrastructural stain morphology is that baseline data on fly stains deposited on materials typical of crime scenes, such as fabrics, metal, glass, or plaster is still scarce [11]. Moreover, the morphologic features of FA related to the activity of a fly may differ from the FA produced from other fly species [12].

The aim of the present study was to employ SEM for the analysis of FA produced by Calliphora vomitoria ( $C$. vomitoria), on five different surfaces that can be commonly present in a crime scene, namely, metal, plaster, glass, cotton, and polyester, in order to obtain further information on the ultrastructural distinction between FA and genuine bloodstains.

\section{Materials and methods}

\section{Scene and experiment}

Scene One hundred adults of $C$. vomitoria were placed in a scaled-down room analog, referred to hereinafter as the "fly box." The fly box was $0.12 \mathrm{~m}^{3}(1 \times 0.3 \times 0.4 \mathrm{~m})$ with five wooden walls and one transparent wall to allow observation. Five different household materials were attached to the fly box walls: glass, plaster, metal, cotton, and polyester. Fifty $\mathrm{mL}$ of fresh human blood with EDTA were placed in a 0.008 $\mathrm{m}^{3}$ box on the floor of the fly box and were used as a blood reservoir for blowflies.

Experiment The blowflies were placed in the fly box for $48 \mathrm{~h}$, and, after that period, the fly box was opened, and the household materials covering the walls were removed. Twenty-five FA, five for each type of material, were sampled and analyzed macroscopically and via SEM.

Control sample The scene was re-arranged as previously described, but in this case, the fresh human blood placed in a $0.008 \mathrm{~m}^{3}$ box on the floor of the box was used to create a parent stain [13]. The parent bloodstain was shot through a cylindrical plastic stick until bloodstains were produced on the walls of the box. Five bloodstains with a diameter lower than $0.3 \mathrm{~cm}$ were randomly sampled from each type of surface and were used as controls.

\section{Experiment with defrosted blood}

The absence of red blood cells in FA, which were always present in bloodstains, was one of the distinctive features observed in the previous study. In forensic casework, when the corpse is removed after longer PMI, it may be necessary to distinguish between spots of hemolyzed blood and FA produced from this source. In order to test the morphology of FA produced from hemolyzed blood, the scene and the experiment were repeated using fresh defrosted human blood taken from a living donor and preserved at $-20^{\circ} \mathrm{C}$ for 1 day, as a blood reservoir for blowflies.

\section{Analysis of spots}

Aiming at dividing the FA obtained in the experiment in categories based on gross macroscopic features, all spots were photographed with an Olympus E-520 camera equipped with an Olympus Zuiko Digital 35 mm 1:35 Macro lens. Color, surface, shape of the body, edges, and tail were observed and described for each spot.

After visual analysis, all the spots were prepared for SEM analysis as follows: from each type of material, a little square sample $(1.2 \mathrm{~cm}$ side) surrounding blood drops or FA was withdrawn. All samples were mounted on a suitable gold-palladium-coated stub with carbon substrate. Then the samples were examined (analyzed) at standard low $(20-40 \times)$, medium low $(300-600 \times)$, and high ultrastructural $(1200 \times)$ magnification through a SEM Stereoscan 360, Leica, Cambridge, with electron secondary probe at $15 \mathrm{kV}$, to appreciate not only shape, but morphological 
ultrastructural characteristics such as the appearance of the surface of the spot, the presence of deposits of foreign material, and the spot cell morphology.

\section{Results}

The preliminary macroscopic analysis was aimed at identifying three categories of FA, based on gross visual features of the spots. FA on hard surfaces were divided into dark color FA (DFA), light color FA (LFA) and tailed FA (TFA). The results of visual analysis on hard surfaces are reported in Fig. 1 a-d (glass), m-p (plaster), and y-bb (metal). On cotton and polyester, FA with a tail clearly distinguishable from the body of the spot were absent. The results of visual analysis on fabrics are reported in Fig. 2 a-c (cotton) and $\mathrm{j}-1$ (polyester).

Visual features of FA on hard surfaces (glass, metal, plaster) are reported in Table 1, while visual features of FA on fabrics (cotton and polyester) are reported in Table 2.
Fig. 1 FA deposited on hard surfaces, namely, glass $(\mathrm{a}-\mathrm{l})$, plaster $(\mathrm{m}-\mathrm{x})$, and metal $(\mathrm{j}-\mathrm{jj})$. The figure reports the visual analysis, the low magnification SEM analysis (SEM $1 \mathrm{~m}$ ), and the high magnification SEM analysis (SEM hm) of dark color FA, light color FA, tailed FA, and genuine bloodspots (control)

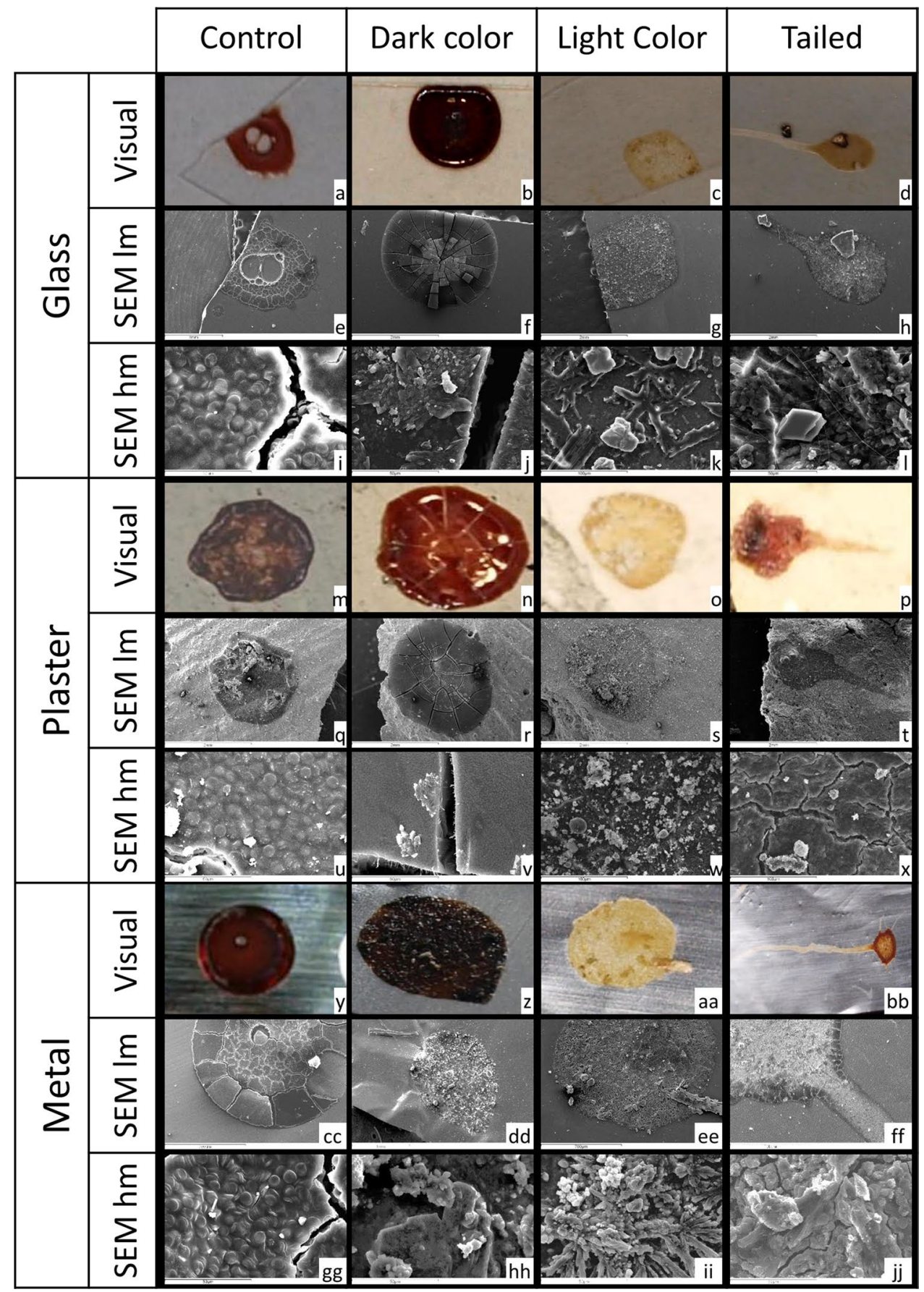




\section{SEM analysis on hard surfaces}

The results of SEM analysis on hard surfaces are reported in Fig. $1 \mathrm{e}-1$ (glass), $\mathrm{q}-\mathrm{x}$ (plaster), and cc-jj (metal).
FA showed on the surface of the small stain deposits, absent on the surface of control samples. Deposits on the surface of FA consisted of amorphous crystals, micro-crystals with morphology similar to those of uric,

Fig. 2 FA deposited on fabrics, namely, cotton (a-i) and polyester (j-r). The figure reports the visual analysis, the low magnification SEM analysis (SEM $1 \mathrm{~m}$ ), and the high magnification SEM analysis (SEM hm) of dark color FA, light color FA, and genuine bloodspots (control)

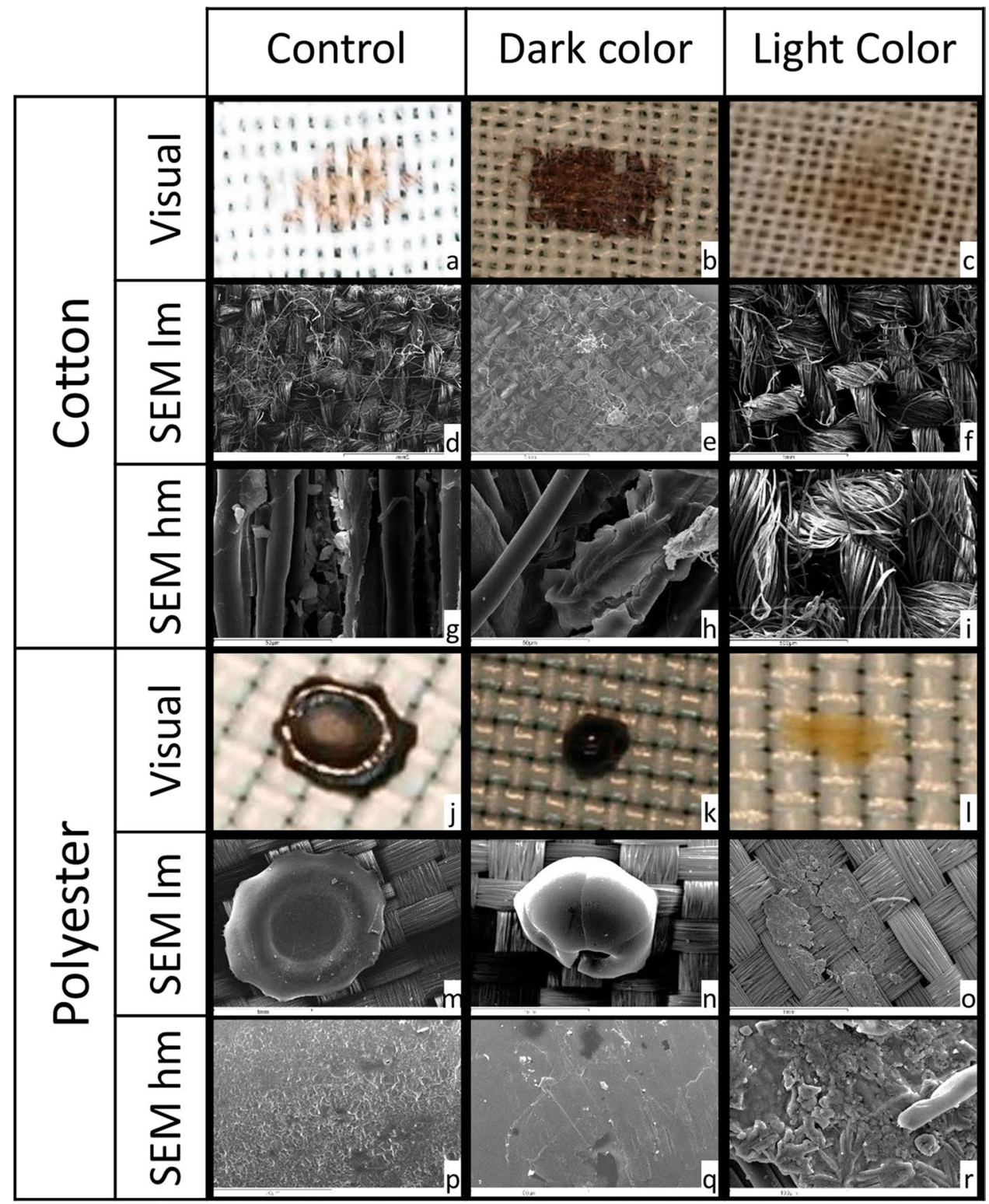

Table 1 Gross visual features of FA on hard surfaces (glass, metal, polyester)

\begin{tabular}{|c|c|c|c|c|}
\hline & Dark fly artifacts (DFA) & Light fly artifacts (LFA) & Tailed fly artifacts (TFA) & Controls (bloodspots) \\
\hline Color & Red/brownish & Yellow/light brown & $\begin{array}{l}\text { Red/brownish or yellow/light } \\
\text { brown }\end{array}$ & Red/brownish \\
\hline Shape of the body & Circular or elliptical & & & \\
\hline Surface & Flat, cratered, or textured & & Textured & Flat or cratered \\
\hline Edges & Linear or slightly scalloped & & & \\
\hline Tail & $\begin{array}{l}\text { Absent or shorter than the } \\
\text { body }\end{array}$ & $\begin{array}{l}\text { Absent or shorter than the } \\
\text { body }\end{array}$ & Longer than the body & $\begin{array}{l}\text { Absent or shorter than the } \\
\text { body }\end{array}$ \\
\hline
\end{tabular}


Table 2 Gross visual features of FA on fabrics (cotton and polyester)

\begin{tabular}{llll}
\hline & Dark fly artifacts (DFA) & Light fly artifacts (LFA) & Controls (bloodspots) \\
\hline Color & Red/brownish & Yellow/light brown & Red/brownish \\
Shape of the body & Circular or elliptical & Circular, elliptical, or linear & Circular or elliptical \\
Surface (cotton) & Reproduced the texture of the underlying cotton fabric & & Flat or cratered \\
Surface (polyester) & Flat or cratered & Flat or textured & \\
Edges (cotton) & Reproduced the underlying texture of the fabric & \\
Edges (polyester) & Linear, sometimes slightly scalloped & \\
Tail & Absent or shorter than the body & & \\
\hline
\end{tabular}

or micro-crystals with morphology similar to those of cholesterol.

Red blood cells (RBC) were absent in FA, while the surface of the controls was paved by RBC appreciable as biconcave discs, maintaining their own shape and appearing stacked in "rouleaux" due to drying fixation.

FA produced from defrosted hemolyzed blood showed crystals structurally similar to those previously observed. As expected, RBC were absent both in FA and in controls.

\section{SEM analysis on fabrics}

The results of SEM analysis on fabrics are reported in Fig. 2 $\mathrm{d}-\mathrm{i}$ (cotton) and $\mathrm{m}-\mathrm{r}$ (polyester).

On cotton, for both FA and controls, the drop was almost completely absorbed and thus indistinguishable from the underlying texture of fabrics. Deposits and RBC were not visible both on FA and on controls.

On polyester, the surface of DFA was completely flat and sometimes cratered and was not explorable with SEM. Deposits and RBC on the surface of DFA and controls were not visible. The surface of LFA was glomerular. Deposits on the surface of LFA consisted of amorphous crystals and micro-crystals with morphology similar to those of uric or cholesterol. RBC were absent in LFA.

FA produced from defrosted hemolyzed blood showed crystals structurally similar to those previously observed. As expected, $\mathrm{RBC}$ were absent both in FA and in controls.

\section{Discussion}

FA can display an innumerable variety of shapes due to the nature and number of variables that contribute to their formation, such as the different behaviors of fly species and the different deposition surfaces. Although studies have tried to produce FA under different experimental conditions on many surfaces, no complete consensus of physical attributes of FA has been reported [3, 8, 11, 14].

Durdle et al. [7] reported some specific features that improved the identification of FA at the crime scene based on their visual morphology. Following a rigorous scientific visual approach, most pitfalls related to classification can be avoided, especially when multiple stains are present and consequently specific features can be compared between different spots, such as the presence of a parent stain, the directionality, the color or shade of colors, the impact angle, and size $[3,15]$. However, some types of FA, such as isolated dark red spots, are also difficult to distinguish from a bloodstain for educated and experienced pathologists and criminalists [15]. Moreover, experts in this distinction do not always attend crime scenes [14]. A confirmatory assay for the identification of FA is needed, especially in uncertain scenarios, where spots are of indeterminate origin also after a careful visual analysis.

The present study was developed to analyze FA produced by $C$. vomitoria on different surfaces that are commonly part of a crime scene. In particular, the aim of our investigation was to test the potential utility of SEM for distinguishing bloodstains from FA.

The preliminary macroscopic analysis was carried out to cluster FA into categories, namely, DFA, LFA, and TFA, on hard surfaces and DFA and LFA on fabrics. The absence of TFA on fabrics may be related to the particular behavior of stains on the array of textiles. In point of fact, when found on clothing apparel, the appearance of stains depends on fabric absorbency and texture, fabric construction (e.g., yarn size, twist level, fabric weight), and wear condition [16-19], as well as characteristics of the fluid itself, such as volume and composition [20].

On hard surfaces, at ultrastructural SEM analysis, a distinctive feature of FA was the presence at high magnification of amorphous crystals and/or micro-crystals with morphology similar to those of uric, which have been described in defecatory FA [4, 21], or cholesterol. Moreover, FA did 
Fig. 3 Flow chart depicting how a fly artifact can be distinguished from a blood spot using SEM ultrastructural analysis

\section{Ultrastructural SEM analysis}
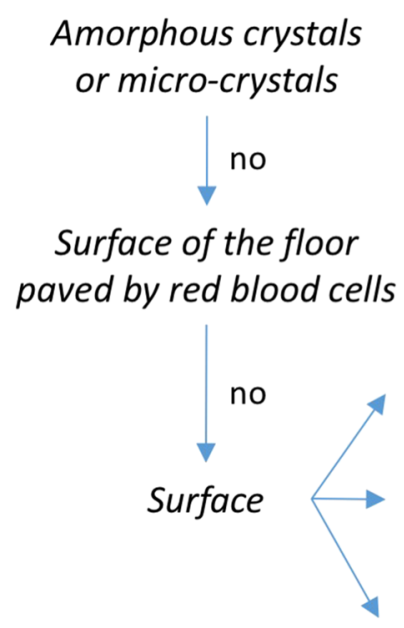

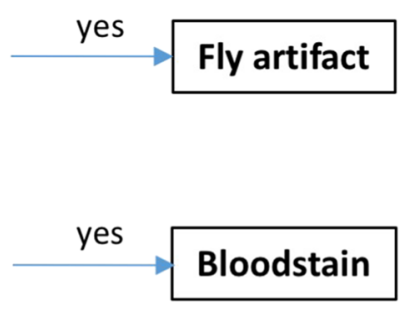

Exporable

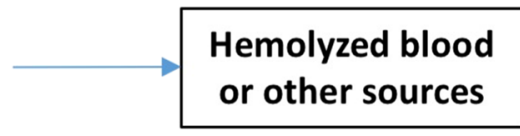

Flat unexporable

\section{Absorbed}

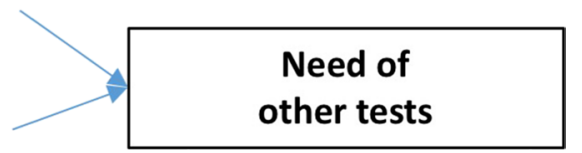

not present RBC, while on controls, the surface of the stain was paved by RBC. These results were in agreement with the features observed on FA deposited by S. carnaria on paper [10].

In order to test the hypothesis of a correlation of the amorphous crystals and/or micro-crystal deposits with RBC degradation, the experiment was repeated analyzing FA produced by flies from defrosted hemolyzed blood. Furthermore, the presence of deposits on FA produced from hemolyzed blood support the hypothesis that deposits may be produced during the digestive activity of flies.

In forensic casework, for bloodstains that could originate from hemolyzed blood when the corpse is moved after longer PMI, the absence of RBC on the spot could lead to erroneous interpretation. To be correctly qualitatively identified as a FA, the stain should present the deposits described in the present study that were always present in all FA deposited on hard surfaces.

On fabrics, SEM analysis was cumbersome. In fact, on cotton, the complete absorption of the stain, which was appreciable also macroscopically, confounded the ultrastructural analysis. On both controls and FA, it was only possible to observe non-specific residues of biological material that did not display the features of RBC, neither of the amorphous nor crystal-like material.

On polyester, the surface of DFA and controls was completely flat. Consequently, the beam of accelerated electrons could not penetrate the surface of the bloodspot because of the absence of superficial cracks and it was therefore impossible to appreciate RBC neither to amorphous/crystal-like material and to make a differential diagnosis.
The surface of LFA displayed uric/cholesterol-like and amorphous deposits; RBC were absent, as observed on hard surfaces, allowing differential diagnosis.

Based on the results obtained from the present study, we report in Fig. 3 a flow chart of the potential application of SEM ultrastructural analysis. In some cases, the origin of the stain can remain undetermined even after a combined macroscopic ultrastructural approach, such as in the case of darkcolored stains with a flat surface that cannot be explored by SEM, like those we observed on polyester, or stains absorbed by the deposition surface, like those we observed on cotton. In these cases, other non-morphological techniques could be used, such as the recently developed immunodetection with polyclonal antiserum [11, 12, 14, 22]. Even if validation studies are needed on other experimental settings, SEM analysis confirmed to be a promising tool for distinctions of FA from true human bloodstains.

The presented method has some unavoidable limitations, due to the technique of analysis and the type of instrument used. First, SEM is not readily available to all forensic medicine services, being an expensive technique that sees few and specific forensic applications. In any case, the forensic experts who intervene during the crime scene investigation must be aware of the possibility of using this technique in the case of macroscopically indistinguishable spots, as an integrative technique to those already proposed in the literature. Furthermore, the preparation of the sample involves an irreversible modification of the spot, which cannot be used for further analysis. Therefore, SEM analysis must be performed as a last resort, after resorting to other more conservative approaches. Another issue is related to the phase of sampling, since for the analysis, it is necessary to sample not only the spot, but also the underlying substrate, such as 
the flash of a window or a part of plaster. This implies an irreversible modification of the crime scene. For this reason, we recommend the sampling for SEM analysis following an accurate high-resolution photographic collection of the spots, according to shared procedures [3].

\section{Conclusions}

As previously observed on FA deposited by $S$. carnaria on different types of paper, SEM analysis, through the investigation of surface deposits and RBC, was confirmed as suitable for differential diagnosis on hard surfaces such as metal, glass, and plaster. On fabrics, namely, cotton and polyester, SEM analysis may be inconclusive. When it is necessary to identify the origin of an ambiguous stain and insect activity is suspected, a multidisciplinary approach to bloodstain pattern analysis is strongly suggested, including SEM analysis.

Acknowledgements Authors would like to kindly thank Luigi Ruggeri for breeding and supplying pupae and adult flies of $C$. vomitoria.

Funding Open access funding provided by Alma Mater Studiorum Università di Bologna within the CRUI-CARE Agreement.

\section{Declarations}

Ethics approval All procedures performed in this study were in accordance with the ethical standards of the national research committee (Bioethics Committee of the University of Bologna, Prot. 55,171, 18/03/2019).

Consent to participate As fresh blood was used in this study, informed consent was obtained from all donors.

Conflict of interest The authors declare no competing interests.

Open Access This article is licensed under a Creative Commons Attribution 4.0 International License, which permits use, sharing, adaptation, distribution and reproduction in any medium or format, as long as you give appropriate credit to the original author(s) and the source, provide a link to the Creative Commons licence, and indicate if changes were made. The images or other third party material in this article are included in the article's Creative Commons licence, unless indicated otherwise in a credit line to the material. If material is not included in the article's Creative Commons licence and your intended use is not permitted by statutory regulation or exceeds the permitted use, you will need to obtain permission directly from the copyright holder. To view a copy of this licence, visit http://creativecommons.org/licenses/by/4.0/.

\section{References}

1. Benecke M, Josephi E, Zweihoff R (2004) Neglect of the elderly: forensic entomology cases and considerations. Forensic Sci Int
146(Suppl):S195-S199. https://doi.org/10.1016/j.forsciint.2004. 09.061

2. Benecke M, Lessig R (2001) Child neglect and forensic entomology. Forensic Sci Int 120:155-159. https://doi.org/10.1016/ s0379-0738(01)00424-8

3. Viero A, Montisci M, Pelletti G, Vanin S (2019) Crime scene and body alterations caused by arthropods: implications in death investigation. Int J Legal Med 133:307-316. https://doi.org/10. 1007/s00414-018-1883-8

4. Rivers D, Geiman T (2017) Insect artifacts are more than just altered bloodstains. Insects 8:37. https://doi.org/10.3390/insec ts8020037

5. Benecke M, Barksdale L (2003) Distinction of bloodstain patterns from fly artifacts. Forensic Sci Int 137:152-159. https:// doi.org/10.1016/j.forsciint.2003.07.012

6. Durdle A, van Oorschot RAH, Mitchell RJ (2009) The transfer of human DNA by Lucilia cuprina (Meigen) (Diptera; Calliphoridae). Forensic Sci Int: Genet Suppl Series 2:180-182. https://doi.org/10.1016/j.fsigss.2009.08.095

7. Durdle A, van Oorschot RA, Mitchell RJ (2013) The morphology of fecal and regurgitation artifacts deposited by the blow fly Lucilia cuprina fed a diet of human blood. J Forensic Sci 58:897-903. https://doi.org/10.1111/1556-4029.12145

8. Striman B, Fujikawa A, Barksdale L, Carter DO (2011) Alteration of expirated bloodstain patterns by Calliphora vicina and Lucilia sericata (Diptera: Calliphoridae) through ingestion and deposition of artifacts. J Forensic Sci 56(Suppl 1):S123-127. https://doi.org/10.1111/j.1556-4029.2010.01575.x

9. Langer SV, Illes M (2015) Confounding factors of fly artefacts in bloodstain pattern analysis. J Can Soc Forensic Sci 48:215224. https://doi.org/10.1080/00085030.2015.1083306

10. Pelletti G, Mazzotti MC, Fais P, Martini D, Ingrà L, Amadasi A, Palazzo C, Falconi M, Pelotti S (2019) Scanning electron microscopy in the identification of fly artifacts. Int J Legal Med 133:1575-1580. https://doi.org/10.1007/s00414-019-02090-5

11. Rivers DB, Dunphy B, Hammerschmidt C, Carrigan A (2020) Characterization of insect stains deposited by Calliphora vicina (Diptera: Calliphoridae) on shirt fabrics. J Med Entomol 57:1399-1406. https://doi.org/10.1093/jme/tjaa052

12. Rivers DB, Cavanagh G, Greisman V, Brogan R, Schoeffield A (2020) Detection of fly artifacts from four species of necrophagous flies on household materials using immunoassays. Int J Legal Med 134:1239-1253. https://doi.org/10.1007/ s00414-019-02159-1

13. Scientific Working Group on Bloodstain Pattern Analysis (SWGSTAIN) (2009) Scientific working group on bloodstain pattern analysis: recommended terminology. https://archives.fbi.gov/archi ves/about-us/lab/forensic-science-communications/fsc/april2009/ standards/2009_04_standards01.htm. Accessed 17 Mar 2021

14. Rivers DB, Cavanagh G, Greisman V, Brogan R, Schoeffield A, McGregor A (2019) Response to "Commentary on: Rivers DB et al. Immunoassay detection of fly artifacts produced by several species of necrophagous flies following feeding on human blood. Forensic Science International: Synergy 2019;1(1):1-10." Forensic Sci Int 1:305-306. https://doi.org/10.1016/j.fsisyn.2019. 04.006

15. Ristenbatt RR 3rd (2019) Commentary on: Rivers DB et al. Immunoassay detection of fly artifacts produced by several species of necrophagous flies following feeding on human blood. Forensic Science International: Synergy 2019;1(1):1-10. Forensic Sci Int 1:303-304. https://doi.org/10.1016/j.fsisyn.2019.04.007 
16. White B (1986) Bloodstain patterns on fabrics: the effect of drop volume, dropping height and impact angle. J Can Soc Forensic Sci 19:3-36. https://doi.org/10.1080/00085030.1986.10757399

17. Slemko JA (2003) Bloodstains on fabric: the effects of droplet velocity and fabric composition. IABPA News 19:3-11

18. de Castro TC, Taylor MC, Kieser JA, Carr DJ, Duncan W (2015) Systematic investigation of drip stains on apparel fabrics: the effects of prior-laundering, fibre content and fabric structure on final stain appearance. Forensic Sci Int 250:98-109. https://doi. org/10.1016/j.forsciint.2015.03.004

19. Williams EM, Dodds M, Taylor MC, Li J, Michielsen S (2016) Impact dynamics of porcine drip bloodstains on fabrics. Forensic Sci Int 262:66-72. https://doi.org/10.1016/j.forsciint.2016.02.037

20. Holbrook M (2010) Evaluation of blood deposition on fabric: distinguishing spatter and transfer stain. IABPA News 26:3-12
21. Wigglesworth VB (1972) The principles of insect physiology. New York: Chapman and Hall Ltd

22. Rivers DB, Cavanagh G, Greisman V, McGregor A, Brogan R, Schoeffield A (2018) Immunoassay detection of fly artifacts produced by several species of necrophagous flies following feeding on human blood. Forensic Sci Int 1:1-10. https://doi.org/10. 1016/j.fsisyn.2018.11.001

Publisher's note Springer Nature remains neutral with regard to jurisdictional claims in published maps and institutional affiliations. 